\title{
Certificates of Recoverability with Scalable Recovery Agent Security
}

\author{
Eric R. Verheul \\ PricewaterhouseCoopers, GRMS Crypto Group, P.O. Box 85096, 3508 AB Utrecht \\ Eric.Verheul@[nl.pwcglobal.com, pobox.com]
}

\begin{abstract}
We propose new schemes for Certificates of Recoverability (CRs). These consist of a user's public key and attributes, its private key encrypted in such a way that it is recoverable by one or more Key Recovery Agents (KRAs), plus a publicly verifiable proof of this (the actual CR). In the original schemes, the level of cryptographic security employed by the KRA and the users is necessarily the same. In our schemes the level of cryptographic security employed by the KRA can be set higher, in a scalable fashion, than that being employed by the users. Among the other improvements of our schemes are its applicability to create CRs for cryptosystems based on the Discrete Log problem in small subgroups, most notably the Digital Signature Standard and Elliptic Curve Crypto systems. Also, the size of the constructed proofs of knowledge can be taken smaller than in the original schemes. We additionally show several ways to support secret sharing in our scheme. Finally we present several new constructions and results on the hardness of "small parts", in the setting of Diffie-Hellman keys in extension fields.
\end{abstract}

\section{Introduction}

In a Public Key Infrastructure there are several roles, of which we name:

Participants Who rely on public key encryption and need to be confident that the associated private key is owned by the user mentioned in the public key.

Certification Authorities This confidence is obtained through the use of $p u b$ lic key certificates, which bind public keys and its attributes to a user. The binding is achieved by having a trusted Certification Authority verifying the user's identity and other attributes and then digitally sign each certificate. This verification is usually performed by an optional entity, called Registration Agent "closer to the user" than the CA.

Key Recovery Agents Sometimes a user or the organization the user works for, requires an emergency back-up of its private key and deposits it with a trusted Key Recovery Agent (KRA).

It could be part of a Certification Policy of a user's organization or CA that no certificates are issued unless the associated private key is properly escrowed with a KRA. Of course, the KRA should operate under a strict, well documented, and 
regularly audited, recovery policy. In [27] the concept of Certificate of Recoverability (CR) is introduced which supports such policies. Here a user offers three components to a CA (or RA):

C1 Its public key, identity and other attributes.

C2 An encrypted copy of its private key, decryptable only by one or more Key Recovery Agents.

C3 A publicly verifiable proof that the first two components are correctly formed, i.e. that the private key of the public key in component $\mathbf{C 1}$ coincides with the encrypted private key in component C2. This component is called the Certificate of Recoverability.

After verifying the proof in C3, the CA issues a regular certificate on the public key. In addition, the CA archives the three components or sends them to the KRA(s). In a conventional escrow scheme, i.e. without the component C3, the CA (or RA) would have setup a KRA-acceptance phase. This consists of sending components $\mathbf{C} 1$ and $\mathbf{C 2}$ to all the KRAs to verify the validity of C2. The central advantage of a $\mathrm{CR}$ scheme compared with a conventional escrow system, is that the CA can perform this KRA-acceptance phase without having to communicate with the $\mathrm{KRA}(\mathrm{s})$. This gives rise to the many advantages of a $\mathrm{CR}$ scheme, like speed, cost effectiveness and security.

The publicly verifiable proof in component C3 should preferably have the property of being perfectly zero-knowledge, or being a parallel version of it, see [26]. Also, observe that the user's private key is contained in both his public key and in component C2. So, preferably there should be a guarantee for combined security, i.e. that this combination of encryption schemes (used by users and KRAs) does not result in an exploit, yielding (parts of) the user's private key. Compare [11], where combined (in)security is discussed for the RSA cryptosystem.

In [27] a construction for the CR concept is given, which is related to a construction in [24]. Here a prime number $p$ and a cyclic group $G=\langle g\rangle$ of order $p$ are generated (for instance by the $\mathrm{CA}$ ) in which the discrete logarithm problem is intractable. The idea is that the public keys of users take the form $g^{x}$, where $0<x<p$, is the associated private key.

In addition, the ElGamal encryption scheme [8] in a multiplicative subgroup $\Gamma$ of order $\omega$ in the (basic) finite field $G F(p)$ is used to encrypt a copy of the private key $x$ in C2. The last component C3 consists of a transcript of a perfect zeroknowledge proof of knowledge, proving that the private key $x$ is encrypted in both C1 and C2. Moreover, a guarantee of combined security for this construction is given in [24].

In both [24] and [27] it is suggested to combine the construction of the system parameters of users and KRAs. One starts with choosing the order $\omega$ as a large prime number, such that $p=2 \omega+1$ and $r=2 p+1$ are prime as well. Then take $G$ as the subgroup of order $p$ in the multiplicative group of $G F(r)$ and $\Gamma$ is the subgroup of order $\omega$ in the multiplicative group of $G F(p)$. 
For sufficient security, $r, p, \omega$ should be of sufficient size, of say $\geq 1000$ bits. Also, to obtain a soundness level of $2^{-v}$ in the above scheme, the size of a transcript is roughly $v$ times the number of bits in $p$, e.g. 100,000 bits for $v=100$ and $|p|=1000$.

This type of construction of CRs has two major drawbacks:

1. The level of security given by the KRA's public key is the same as the security given by the public keys of the users. The KRA's private key gives access to all users' private keys, as it is a master key. Hence the KRA's public key is subject to more severe threats than the users' public keys. For instance, attackers wanting access to communication of different users, could form an alliance to break the KRA's public key. It is "best practice" that master keys, such as KRA's public keys, should be considerably more difficult to break than user's keys, i.e. public keys of the users. This is also why the signing key of a (root) Certifying Authority is typically longer than that of its users.

We remark that using stronger KRA's public keys is only sensible, provided that breaking one user's public key does not imply breaking all users' public keys. This is the case when using DL-based schemes. In the setting of a finite field, breaking one discrete logarithm based public key considerably helps in breaking other ones, but that still takes sub-exponential time (cf. [10], [26, p.172]). In the setting of Elliptic Curve Cryptosystems, breaking one public key doesn't help (much) in breaking other public keys: an exhaustive search proportional to the square root of the group's order still has to be conducted.

2. The use of DL-based schemes in groups of relative small order (of say of size 160 bit), can be very beneficiary in terms of speed and data-storage. Such groups are employed in the Digital Signature Standard and in Elliptic Curve Crypto systems. However, the schemes from [27], forces users to use groups of orders of at least 1024 bits to have sufficient security; making it impossible to employ these beneficiary groups.

To remedy these two drawbacks, one could use the generic verifiable encryption scheme that appears in [2]. However, the resulting interactive proof in C3 would only have the property of computational zero-knowledge. Also, no formal guarantee for combined security, as defined above, is given in [2].

Our System We propose new schemes for CRs which do not have the above mentioned drawbacks. In our schemes, users can use any type of cyclic group $G$ of prime order $p$ in which the discrete logarithm is intractable. This means that the size of $p$ should be sufficiently large, of say $\geq 160$ bits; but no further restrictions are made. The user's private key is encrypted for the KRA, using the ElGamal scheme in a field extension $G F\left(p^{t}\right)$. Here one can choose to employ the classical ElGamal scheme, i.e. using the whole multiplicative group of $G F\left(p^{t}\right)$, or the subgroup variant as proposed by Schnorr [23], using a multiplicative subgroup of $G F\left(p^{t}\right)$ of suitable, large prime order.

In both variants the proofs of knowledge, C3, can be formed in a perfect zeroknowledge fashion, the size of which can be taken considerably smaller than that 
from [27] and [24]. Moreover, by employing optimal normal bases our schemes are more efficient than comparable schemes in [27]. Finally, the guarantee for combined security is proven to be given by the hardness of the Diffie-Hellman Decision Problem.

Apart from key-escrow applications, our techniques can be applied in the design of electronic cash systems providing revocable anonymity [25] in which the identity of the system's users can be recovered when the system is abused for criminal activities. The recovery of the identity is done with the help of trustees, whose role can be compared with that of the above mentioned KRAs.

We remark that we do not incorporate the CR (i.e. C3) itself in the issued certificate. There is no need to do so, and in our schemes this would also introduce the possibility of the establishment of shadow public keys, see [12].

Outline of the paper Apart from presenting new schemes for Certificates of Recoverability, we present several new results and constructions in multiplicative subgroups of extension fields. In Section 2 we will first discuss new results on the hardness of "small parts" of the key exchanged in the Diffie-Hellman protocol in extension fields. Section 3 deals with proving knowledge on equality of logarithms in the setting of extension fields. In Section 4, we present our basic scheme, using the results of Sections 2 and 3 to prove security and functionality. In this section we also describe generalizations, like incorporating secret sharing, and efficiency improvements of the scheme.

\section{DL-Based Cryptosystems over Extension Fields}

\subsection{Introduction}

Let $H$ be a multiplicative, cyclic group in which the discrete logarithm problem is intractable and $h$ is a generator of $H$ of order $o$. The $h, H$ and $o$ are system parameters given to all participants. The Diffie-Hellman (DH) key agreement [7] was the first practical solution for allowing two parties to agree over an insecure channel on a common secret key. The security of it lies in the Diffie-Hellman problem of computing values of the function $\mathrm{DH}\left(h^{x}, h^{y}\right)=h^{x y}$. A drawback of the basic DH scheme is that the parties involved, Alice and Bob say, can only share one secret key. This is remedied in several schemes related to the DH scheme, such as the ElGamal encryption scheme [8]. Here Bob picks a random $0<x<o$ and publishes his public key $y=h^{x}$. If Alice wants to send a message $m \in G$ to Bob, then Alice picks a random $0<k<o$ and sends $(A, B)=\left(h^{k}, m \cdot y^{k}\right)$ to Bob. Upon receipt, Bob decrypts the message by forming $B / A^{x}$ which is equal to the message $m$.

Two other problems are related to DH problem. The first one is the DiffieHellman Decision problem: given $a, b, c \in\langle h\rangle$ determine whether $c=\operatorname{DH}(a, b)$. The DH problem is at least as difficult as the DH Decision problem. The second related problem is the Discrete Logarithm (DL) problem in $\langle h\rangle$ is given $a \in\langle h\rangle$, find $0<x<o$ such that $a=h^{x}$. The DL problem is at least as difficult as the 
DH problem. For the security of the Diffie-Hellman scheme, all three problems should be intractable.

We call $h^{\prime}$ a prime subgenerator of $h$ if $h^{\prime} \in\langle h\rangle$ and the order of $h^{\prime}$ is a prime number. By virtue of the Pohlig-Hellman algorithm [17], the difficulty of the DL problem w.r.t. $h$, given the factorization of the order of $h$, is as difficult as solving the DL problem for all prime subgenerators of $h$.

Clearly, one can construct the Diffie-Hellman and related schemes in any multiplicative subgroup $\Gamma=\langle\gamma\rangle$ of $G F\left(p^{t}\right)$ of order $\omega$. For solving the discrete logarithm problem for a prime subgenerator $\gamma^{\prime}$ of $\gamma$ of order $\omega^{\prime}$, one can use an index calculus (IC) based algorithm that has a heuristic expected asymptotic running time of $L\left(p^{s}, 1 / 3,1.923+o(1)\right]$, see [1] and [13], where $s$ is the smallest divisor of $t$ such that $\left\langle\gamma^{\prime}\right\rangle$ is contained in a subfield of $G F\left(p^{t}\right)$ isomorphic to $G F\left(p^{s}\right)$. If $p=2$ then the constant 1.923 can be replaced by 1.587 , see [6]. Alternatively one can use Birthday Paradox (BP) based algorithms (e.g. Pollard's rho algorithm [18]) that have expected running times exponential in the size of the $\omega^{\prime}$. More precisely, breaking the Discrete Logarithm problem can be solved in expected $O\left(\sqrt{\omega^{\prime}}\right)$ elementary operations in $G F\left(p^{t}\right)$.

This leads us to the conclusion from [13] that w.r.t. attacks known today the intractability of the discrete logarithm problem in $\Gamma$ depends on the existence of a prime generator $\gamma^{\prime}$ of $\gamma$ whose minimal surrounding subfield and prime order are of sufficient size. The particular form of the field itself, is not relevant. In other words, if $G F\left(p^{t}\right)$ is the minimal surrounding field of a subgroup of prime order, then - w.r.t. attack known today - the discrete logarithm in this subgroup is approximately as difficult as the discrete logarithm in a subgroup of prime order of a basic field $G F(P)$ if the size of $P$ is approximately equal to as $t$ times the size of $p$, and the order of both subgroups are about the same size.

Hence, a suitable generator $\gamma$ in a field extension $G F\left(p^{t}\right)$ should have a prime subgenerator that is not contained in one of the proper subfields and should have a suitably large order. In [13], A.K Lenstra proposes a simple, practical method for the construction of a field extension and suitable generator generating a relatively small subgroup in it. The idea is that one fixes the size of the prime number $p$ and a number $t$ such that $p^{t}$ is "large" enough. Then one looks for a large prime factor $\varpi$ in the value of the cyclotomic polynomial $\phi_{t}(p)$. The latter can be done using trial divisions with the primes up to, say $10^{5}$; any other reasonable bound or method will do. Finally, one constructs a generator $\gamma$ of order $\varpi$, by looking for an element different from 1 , such that $g^{\left(p^{t}-1\right) / \varpi}=1$. If one factorizes $p^{t}-1$, one can also find a generator of the whole multiplicative group of $G F\left(p^{t}\right)$.

Using the above construction, the size of $\varpi$ is about $\varphi(t) \cdot|p|$ bits (where $\varphi($. is Euler's totient function) which grows as least as fast as $p^{t / \log (\log (t))}$. The complexity of the BP based algorithms grows much faster, than the complexity of the IC based algorithms. So if the size of the surrounding field is large enough to resist the sub-exponential, IC based algorithms, then the order $\varpi$ will usually be large enough "automatically" to resist the BP based algorithms as well. 


\subsection{Partial Security of DL-Based Systems over Extension Fields}

Let $\gamma \in G F\left(p^{t}\right)$ be a generator of a group $\Gamma$ of order $\omega$. In our applications, $\gamma$ is not contained in a genuine subfield of $G F\left(p^{t}\right)$ and $\omega$ is either equal to a large prime number $\varpi$ or to $p^{t}-1$. We start with some well-known notions; if $J$ is an element of $G F(p)[X]$, i.e. a polynomial with coefficients in the basis field $G F(p)$, then for any natural number $i$, the $i$-th coefficient of $J$ is denoted by $[J]_{i}$. If $F=\sum_{i=0}^{t} a_{i} X^{i}$ is an irreducible polynomial of degree $t$ in $G F(p)[X]$, then we can describe the extension field $G F\left(p^{t}\right)$ as $G F(p)[X] /(F)$, i.e. each element $f$ in $G F\left(p^{t}\right)$ can be uniquely written modulo $F$, as a polynomial of degree $<t$. In this setting, for any natural number $i$ less than the degree of $F$, we let the $i$-th coefficient $[f]_{i}$ denote $[f \bmod F]_{i}$.

There are many such representations and some have special properties. For instance, if $t+1$ is also prime and that $p$ is a primitive element modulo $t+1$, then one can use a special irreducible polynomial, the zeros of which form an optimal normal basis. Which such a basis, one can do exponentiation in $G F\left(p^{t}\right)$ even more efficiently than in a basic field of comparable size. See [13], where it is also shown how such pairs $p, t$, and the special irreducible polynomial can be easily generated.

Some constructions, like the coefficients, are dependent of the concrete representation of $G F\left(p^{t}\right)$ one has chosen. Throughout the remainder of this paper we assume that one has chosen an irreducible polynomial $F$, yielding a concrete representation of $G F\left(p^{t}\right)$.

Below we prove that computing coefficients (i.e. "small parts") of the DiffieHellman key, is as difficult as computing the whole key. These results are similar to the ones in [4] where the security of the Most Significant Bits (MSB) of Diffie-Hellman keys in a basic finite field $G F(p)$ is studied.

Theorem 21 We use the above terminology. Given an oracle that computes a coefficient of a Diffie-Hellman key $\gamma^{x y}$ on basis of $\gamma^{x}, \gamma^{y}$, then there exists a polynomial time algorithm that solves the Diffie-Hellman problem in $\Gamma=\langle\gamma\rangle$.

Proof: The function that returns a coefficient of a fixed position less than $t$ is linear and is hence a summing function (see below) by Proposition 23 below. The result now immediately follows from Theorem 24 below.

It follows in particular that any bit of the Diffie-Hellman key in $G F\left(2^{t}\right)$ is as hard to compute as the whole. This offers a more conventional alternative for the new variant of the Diffie-Hellman protocol mentioned in [4] having exactly this property. In our situation, the size of $p$ is not small ( $\geq 160$ bit or more) and we immediately obtain the following corollary, which is crucial for the security of our scheme. This result immediately follows directly from Theorem 21.

Corollary 22 Form, in the above terminology, the public key $\psi=\gamma^{x}$ with private key $0<x<\omega$. Then solving $S \in G F(p)$ from the ElGamal encryption $\left(\gamma^{k}, S \cdot\left[\psi^{k}\right]_{i}\right)$ for some fixed $0 \leq i \leq t-1$ and $1 \leq k \leq \omega$ random, is equivalent to solving the Diffie-Hellman problem in $\Gamma=\langle\gamma\rangle$. 
Theorem 21 is a consequence of a much broader result. Let $n$ be a non-negative number and consider the integers $e_{1}, \ldots, e_{n}$ (the "exponents") and the elements $\lambda_{1}, \ldots, \lambda_{n} \in G F\left(p^{t}\right) \backslash\{0\}$ (the "multipliers") and consider the following summing function $Z():.\langle\gamma\rangle \rightarrow G F\left(p^{t}\right)$ defined by:

$$
Z(\kappa)=\sum_{i=1}^{n} \lambda_{i} \cdot \kappa^{e_{i}} .
$$

The number $n$ is called the degree of the summing function and the number $d=\operatorname{gcd}\left(e_{1}, e_{2}, \ldots, e_{n}, \operatorname{ord}(\gamma)\right)$ is called the order of the summing function. Note that if all multipliers are elements of the basic field $G F(p)$, then its form is representation-independent.

The following result implies that the collection of summing functions is rather large.

Proposition 23 If $f($.$) is a linear mapping from G F\left(p^{t}\right)$ onto $G F(p)$ (i.e. a functional), then the restriction of $f($.$) to \langle\gamma\rangle$ is a summing function of order 1 . Moreover, the multipliers occurring in the definition of summing function can be easily determined. In particular it follows that the restriction to $\langle\gamma\rangle$ of the trace function $\operatorname{Tr}($.$) of G F\left(p^{t}\right)$ onto the base subfield $G F(p)$ defined by (cf. [16]):

$$
\operatorname{Tr}(\alpha)=\alpha+\alpha^{p}+\ldots+\alpha^{\left(p^{t-1}\right)} .
$$

is a summing function of order 1 .

Proof: It is evident that the trace function is a summing function of order 1. From [16, Theorem 2.24] it follows that (the restriction to $\langle\gamma\rangle$ of) any functional is of the form $\alpha \rightarrow \operatorname{Tr}_{K}(\beta \cdot \alpha)$ for some fixed $\beta \in G F\left(p^{t}\right)$ and is hence a summing function of order 1 .

We note that given a functional $f($.$) , of which we assume it can be efficiently$ evaluate in our concrete representation, one can easily compute the associated $\beta$ (and thus the multipliers) from $f(1), f(\gamma), \ldots, f\left(\gamma^{t-1}\right)$ using the following equality:

$$
\left(\begin{array}{cccc}
1 & 1 & \ldots & 1 \\
\gamma & \gamma^{p} & \ldots & \gamma^{p^{t-1}} \\
\vdots & \vdots & \ldots & \vdots \\
\gamma^{t-1} & \gamma^{(t-1) p} & \ldots & \gamma^{(t-1) p^{t-1}}
\end{array}\right) \cdot\left(\begin{array}{c}
\beta \\
\beta^{p} \\
\vdots \\
\beta^{p^{t-1}}
\end{array}\right)=\left(\begin{array}{c}
f(1) \\
f(\gamma) \\
\vdots \\
f\left(\gamma^{t-1}\right)
\end{array}\right)
$$

Recall that $\gamma$ is an element in $\left.G F\left(p^{t}\right)\right)$ that can not be embedded in a proper subfield of $G F\left(p^{t}\right)$, so $\gamma, \gamma^{p}, \ldots, \gamma^{p^{t-1}}$ are all distinct and the above matrix is a non-singular matrix of the Vandermonde type.

Theorem 24 In the above terminology, let $Z($.$) be a summing function of order$ d. Also let $\mathcal{O}$ be an oracle that on basis of any $\gamma^{a}$ and $\gamma^{b}$ computes $Z\left(\gamma^{a b}\right)$. Then there exists a polynomial time algorithm that computes $\gamma^{a b d}$ on basis of $\gamma^{a}$ and $\gamma^{b}$. That is, for $d=1$ there exists a polynomial time algorithm that solves the whole Diffie-Hellman problem in $\langle\gamma\rangle$. 
Proof: Let $V=\gamma^{x}$ and $W=\gamma^{y}$ be any elements of $\langle\gamma\rangle$. With the help of oracle $\mathcal{O}$ one can not only determine $Z\left(\gamma^{x y}\right)$, but also $Z\left(\gamma^{x(y+i)}\right)$ (by giving the oracle the input $V$ and $\left.\gamma^{i} \cdot W\right)$ for $i=0, \ldots, n-1$, where $n$ denotes the order of $Z($.$) .$ This results in the following equality:

$$
\left(\begin{array}{cccc}
1 & 1 & \ldots & 1 \\
V^{e_{1}} & V^{e_{2}} & \ldots & V^{e_{n}} \\
\vdots & \vdots & \ldots & \vdots \\
V^{(n-1) \cdot e_{1}} & V^{(n-1) \cdot e_{2}} & \ldots & V^{(n-1) \cdot e_{n}}
\end{array}\right) \cdot\left(\begin{array}{c}
\lambda_{1} \cdot \gamma^{x y e_{1}} \\
\lambda_{2} \cdot \gamma^{x y e_{2}} \\
\vdots \\
\lambda_{n} \cdot \gamma^{x y e_{n}}
\end{array}\right)=\left(\begin{array}{c}
Z\left(\gamma^{x y}\right) \\
Z\left(\gamma^{x(y+1)}\right) \\
\vdots \\
Z\left(\gamma^{x(y+n-1}\right)
\end{array}\right)
$$

The above matrix is of the Vandermonde type. Let us first consider the case that all $V^{e_{1}}, V^{e_{2}}, \ldots, V^{e_{n}}$ are all different. Then this matrix is regular, and so from equality (2) one can determine the elements $\gamma^{x y \cdot e_{1}}, \gamma^{x y \cdot e_{2}}, \ldots \gamma^{x y \cdot e_{n}}$. From this one can determine the element $\gamma^{x y d}$ by taking a suitable combination of the $\gamma^{x y \cdot e_{1}}, \gamma^{x y \cdot e_{2}}, \ldots \gamma^{x y \cdot e_{n}}$.

To prove the general case, observe that if $V^{e_{i}}$ and $V^{e_{j}}$ are equal then so are $\gamma^{x y e_{i}}$ and $\gamma^{x y e_{j}}$. So by restricting to a maximal collection of different $V^{e_{i}}$, one can determine $\gamma^{x y d}$ by the above argument.

We again use the above terminology. An element $\omega=\gamma^{x} \in\langle\gamma\rangle$ can also be described in terms of its minimal polynomial of degree $t$. This representation is not unique, as all conjugates of $\omega$, i.e. $\omega, \omega^{p}, \omega^{p^{2}}, \ldots, \omega^{p^{t-1}}$, have the same minimal polynomial. The $i$-th order coefficient of this polynomial equals

$$
(-1)^{i} S_{i}\left(\omega, \omega^{p}, \omega^{p^{2}}, \ldots, \omega^{p^{t-1}}\right),
$$

where $S_{i}$ denotes the elementary symmetric polynomial in $t$ variables of degree $i$. One can easily prove that expression (3) yields a summing function. By virtue of [13, Lemma 2.4] it follows that for $1 \leq i \leq t-1$, the order of this summing function can not contain prime factors $\geq i$. That is, the order of this summing function is very small. Hence it follows from Theorem 24 that for $1 \leq i \leq t-1$, determining an $i$-th order coefficient of the minimal polynomial of the DiffieHellman key, is as difficult as determining the whole key.

Note that one can easily determine the minimal polynomial of $\gamma^{x y}$ on basis of the minimal polynomial of either $\gamma^{x}$ and $y$ or the minimal polynomial of $\gamma^{y}$ and $x$. On this idea variants of the Diffie-Hellman scheme can be based in which the involved parties send each other the minimal polynomials $\gamma^{x}, \gamma^{y}$ rather than the elements themselves. The exchanged secret is the minimal polynomial of the element $\gamma^{x y}$, or some of the non-zero order coefficients of this. More generally one can take the value of a summing function of low order, evaluated in $\gamma^{x y}$. It easily follows from Theorem 24 that such variants are as at least as secure as the original Diffie-Hellman scheme in our concrete representation of $\langle\gamma\rangle$. As minimal polynomials are representation-independent it directly follows that breaking such a variant means breaking the original Diffie-Hellman scheme in any representation of $\langle\gamma\rangle$. The converse, which is less interesting for the security of our variant, is also true but its proof is not elementary. The proof follows by determining zeros of the exchanged minimal polynomials in a fixed representation, 
which can be done in deterministic polynomial time by the technique of H.W. Lenstra Jr. in [15].

The above idea is used in [5] to construct variants of the Diffie-Hellman scheme in which the number of bits exchanged is only a third of what is normally used, while the offered security against attacks known is the same.

Thus far, we have only discussed the unlikeliness that non-noisy oracles exist, that always give correct answers. Now we will briefly discuss the possible existence of noisy oracles $\mathcal{O}$ that on basis of uniformly random $\gamma^{x}$ and $\gamma^{y}$ in $\langle\gamma\rangle$ compute $Z\left(\gamma^{x y}\right)$, with non-negligible probability $\epsilon$. In the general context nonnegligible means that $1 / \epsilon$ is less than a polynomial in $\log _{2}\left(\left|G F\left(p^{t}\right)\right|\right)=t \cdot \log _{2}(p)$. A first indication that such oracles do not exist follows from the easily verified observation that with such an oracle one can solve the Diffie-Hellman Decision problem in $\langle\gamma\rangle$, that is considered hard. See [19].

In our context (the scheme in section 4) it's more natural to say that a probability $\epsilon$ is non-negligible if $1 / \epsilon$ is less than a polynomial in $\log _{2}(p)$. Indeed, a (small) parameter $t$ is designed and then fixed; only the parameter $p$ then varies. We have a heuristic proof for the following variant of Theorem 24 (of which similar variants of Proposition 21 and Corollary 22 can be easily deduced).

Theorem 25 Let $Z($.$) be a summing function on \Gamma$ of degree 1. Given a (noisy) oracle $\mathcal{O}$ that on basis of uniformly random $\gamma^{x}$ and $\gamma^{y}$ computes $Z\left(\gamma^{x y}\right)$, with non-negligible probability $\epsilon$ then there exists a probabilistic polynomial time algorithm that solves the Diffie-Hellman problem in $\langle\gamma\rangle$.

\section{Double Deckers in Field Extensions}

From now on we let $G=\langle g\rangle$ be a cyclic group of order prime order $p$ in which the discrete problem is intractable. We introduce a new notion, that combines $G, G F(p)[X]$, and $G F\left(p^{t}\right)$.

For a polynomial $J$ in $G F(p)[X]$ we define the power polynomial, $g^{J}$ by

$$
\sum_{i=0}^{\operatorname{deg}(J)} g^{[J]_{i}} X^{i} .
$$

Similar to the convention used for regular polynomials, coefficients of powers that are not mentioned are assumed to be equal to the "zero element", which is $g^{0}=1$. $J$ is called the exponent polynomial of $g^{J}$. If $F=\sum_{i=0}^{t} a_{i} X^{i}$ is an (irreducible) polynomial of degree $t$ in $G F(p)[X]$, then we obtain a natural equivalence on the power polynomials: two power polynomials are equivalent iff the difference of their exponent polynomials is a multiple of $F$; the resulting equivalence group is called the group of power polynomials modulo $F$. It is clear that we can represent power polynomials modulo $F$ as $t$ tuples. The collection of power polynomials, resp. power polynomials modulo a polynomial $F$ of degree $t$ will be denoted by $\mathcal{E}$, resp. $\mathcal{E} /(F)$. 
Let $P_{1}=\sum_{i=0}^{m} U_{i} X^{i}$ and $P_{2}=\sum_{i=0}^{n} V_{i} X^{i}$ be two power polynomials. Then the product of $P_{1} \cdot P_{2}$ is defined as the power polynomial $\sum_{i=0}^{\max (m, n)} U_{i} \cdot V_{i} X^{i}$ The inverse $P_{1}^{-1}$ of $P_{1}$ is defined as $\sum_{i=0}^{m} U_{i}^{-1} X^{i}$. If $P_{1}=g^{A}$ and $P_{2}=g^{B}$, then it follows that the $P_{1} \cdot P_{2}=g^{A+B}$ and $P_{1}^{-1}=g^{-A}$. Furthermore, if $C=\sum_{j=0}^{r} c_{j} X^{j}$ is a polynomial in $G F(p)[X]$, then $P_{1}^{C}$ is defined as $\sum_{k=0}^{k=m+r} \prod_{i+j=k} U_{i}^{c_{j}} X^{k}$. It follows that if $P_{1}=g^{A}$, that then $P_{1}^{C}$ is equal to $g^{A \cdot C}$.

Proposition 31 Let $P_{1}=g^{A}=\sum_{i=0}^{m} U_{i} X^{i}$ be a power polynomial of degree $m$, and let $F$ be an (irreducible) polynomial of degree $t$ in $G F(p)[X]$. Then (without knowing $A$ ) one can determine $g^{A \bmod F}$.

Proof: For a proof, let $F=\sum_{i=0}^{t} f_{i} X^{i}$, with $f_{t} \neq 0$. Observe that

$$
g^{\left(A-\frac{a_{m}}{f_{t}} \cdot F \cdot X^{m-t}\right.}=g^{A} \cdot\left(U_{m}\right)^{-f_{t}^{-1} F \cdot X^{m-t}} .
$$

which is a power polynomial of degree $<m$, equivalent with $P_{1}$ modulo $F$ and constructible without knowledge of $A$. The proposition now follows from an inductive argument.

As we remarked before, we represent any $p$-ary polynomial modulo an irreducible $p$-ary polynomial $F$ of degree $t$ (i.e. an element in $G F\left(p^{t}\right)$ ) as a $p$-ary polynomial of degree $<t$. We will also represent any $p$-ary power polynomial modulo $F$ as one of degree $<t$. By the virtue of Proposition 31 we can determine this representation without explicitly knowing the exponent polynomial.

As before, we let $\gamma$ be an element of a multiplicative group of $G F\left(p^{t}\right)$, that is not contained in a proper subfield of $G F\left(p^{t}\right)$ and let $\psi \in \Gamma=\langle\gamma\rangle$. Also, let the order of $\gamma$ be denoted by $\omega$.

Now, suppose that person $P$ (for prover) gives the elements $\mu \in G F\left(p^{t}\right)$ and $M \in \mathcal{E}$ to person $V$ (for verifier) and states:

Assertion DD: There exists a number $k$ less than $\omega$ such that

$$
\mu=\gamma^{k} \quad M=g^{\left(\psi^{k}\right)}
$$

The following protocol, a variant of the double decker protocol in [24], lets $P$ prove statement DD without revealing anything about $k$ or $\psi^{k}$.

\section{Protocol 32}

Pr-1 The Prover generates a random number l less than the order $\omega$ of $\gamma$, calculates $\nu=\gamma^{l}, N=g^{\left(\psi^{l}\right)}$ and hands $\nu$ and $N$ over to $V$.

Pr-2 $V$ generates a random $h \in\{0,1\}$, and presents $h$ as a challenge to $P$.

Pr-3 $P$ calculates $z=l-h \cdot k(\bmod \omega)$ and hands $z$ over to $V$.

Pr-4 $V$ verifies that both

Pr-4a. $\nu=\gamma^{z} \cdot \mu^{h}$, and

Pr-4b. $N=g^{\left(\psi^{z}\right)}$ if $h=0$ and $N=M^{\left(\psi^{z}\right)}$ if $h=1$.

The protocol satisfies the following properties which are easily verified. 
Completeness If statement DD is true, then $V$ will accept it.

Soundness If L-E is not true, then with a probability less than $1 / 2$ it will be accepted by $V$.

Security If L-E is true, then $V$ can not learn any secret information on $k$ or $\psi^{k}$ by following the protocol.

Using the Fiat-Shamir heuristic [9] one can convert Protocol 32 in a non-interactive scheme with knowledge error $2^{-v}$ for a security parameter $v$. To this end, let $H($.$) be a secure hash function with output length equal to v$ bits. For $i=1, \ldots, v$ the Prover chooses $0<l_{i}<\omega$ randomly en calculates (as in Pr-1 above) $\nu_{i}=\gamma^{l_{i}}$ and $N_{i}=g^{\left(\psi^{l_{i}}\right)}$. Then he computes the $v$-tuple

$$
Z=\left(z_{1}, \ldots, z_{v}\right)=\left(l_{1}-h_{1} \cdot k \quad(\bmod \omega), \ldots, l_{v}-h_{v} \cdot k \quad(\bmod \omega)\right)
$$

where $h_{i}$ denotes the $i$-th bit of $H=H\left(\mu, M, \nu_{1}, N_{1}, \ldots, \nu_{v}, N_{v}\right)$. The noninteractive proof consists of $Z$ and $H$. To verify, for $i=1, \ldots, v$, one re-constructs $\nu_{i}, N_{i}$ by:

$$
\begin{aligned}
\nu_{i} & =\gamma_{i}^{z} \cdot \mu^{h_{i}} \\
N_{i} & = \begin{cases}g^{\left(\psi^{z}\right)} & \text { if } h_{i}=0 \\
M^{\left(\psi^{z}\right)} & \text { if } h_{i}=1\end{cases}
\end{aligned}
$$

and then verifies that $H\left(\mu, M, \nu_{1}, N_{1}, \ldots, \nu_{v}, N_{v}\right)$ equals $H$. The size of the noninteractive proof is about $\omega \cdot v$ bits.

As is also explained in [3], a (dishonest) Prover could try to guess the $v$ bits, $h_{i}$, and then form the accompanying responses $z_{i}$. If the Prover's ability to try this is bound by $K$ trials, then the probability of failure of the non-interactive proof equals $K \cdot 2^{-v}$. The choice of $v$ should take this into account. In [3] it is indicated that for a probability of failure less then $2^{-w}$, one should take $v=2 w$, that is $K=2^{v}$. However, this is a correct asymptotic choice and rather arbitrary in practice.

\section{Our Scheme}

\subsection{The Basic Scheme}

As before, let $G=\langle g\rangle$ be a multiplicative, cyclic group of prime order $p$ in which the discrete logarithm problem is intractable. We will not further specify $G$, but in a typical example $G$ is the multiplicative group of a finite field or the group of points on an elliptic curve over a finite field. The $g, G$ and $p$ are system parameters that are not yet fixed; only the size $|p|$ of $p$ is fixed. As in the scheme of [27], we will generate $g, G, p$ until we find one for which we can construct a suitable KRA encryption scheme (see below). The user's public key will be of the form $y=g^{x}$ where $0<x<p$ is the user's private key.

Our security objective is to design an asymmetric cryptosystem based on the Discrete Logarithm problem in a finite field that has "asymmetric security" of 
$D$ bits, e.g. $D \geq 1024$. To this end, choose a number $t$ such that $t \cdot|p| \geq D$. Next, construct random (user) system parameters $g, G, p$ such that $p$ is of size $t$. Next look for a large enough prime number $\varpi$ in the value of the cyclotomic polynomial $\phi_{t}(p)$ (see Section 2), to support the security objective. If this fails, generate new random (user) system parameters $g, G, p$. Next, generate $\gamma$ in $G F\left(p^{t}\right)$ of order $\omega$ either equal to $p^{t}-1$ or $\varpi$. For the first generation, a factorization of $p^{t}-1$ is required. So $\gamma$ either generates the whole multiplicative group of $G F\left(p^{t}\right)$, or a subgroup of order $\omega$. In either case, the Discrete Logarithm is infeasible w.r.t. attacks known today (see Section 2).

Both options correspond with the two types of ElGamal encryptions (classical and subgroup based) the KRA can use, each leading to a different scheme for CRs. In either case, the KRA's public key will be of the form $\psi=\gamma^{x_{K}}$ where $0<x_{K}<\omega$ is the KRA's private key.

Both schemes actually support CRs for a variable number $s$ (with $1 \leq s \leq t$, of different public/private keys $y_{s-1}, \ldots, y_{0} \in G$ owned by the same user (e.g. supporting different applications or Certification Policies) simultaneously. So, by taking $s=1$ in the below schemes we get a Certificate of Recoverability as defined by Young and Yung, for higher $s$ we get a multiple version of this.

Scheme 41 The user generates his $s$ private keys, $x_{0}, \ldots, x_{s-1}$ and forms the following three components:

C1 The user's public keys $y_{s-1}=g^{x_{s-1}}, y_{s-2}=g^{x_{s-2}}, \ldots, y_{1}=g^{x_{1}}, y_{0}=g^{x_{0}}$, his identity and other attributes.

C2 The $s+1$ tuple:

$$
\left(A, B_{s-1}, B_{s-2}, \ldots, B_{0}\right)=\left(\gamma^{k}, x_{s-1}^{-1} \cdot\left[\psi^{k}\right]_{s-1}, x_{t-2}^{-1} \cdot\left[\psi^{k}\right]_{s-2}, \ldots, x_{0}^{-1} \cdot\left[\psi^{k}\right]_{0}\right),
$$

where $0<k<\omega$ is randomly chosen by the user.

C3 A transcript proving that the $x_{s-1}, x_{s-2}, \ldots, x_{1}, x_{0}$ appearing in component $\mathbf{C 1}$, are also appearing in component $\mathbf{C 2}$, i.e. are recoverable from it by the $K R A$.

Observe that, provided that the components $\mathbf{C} 1$ and $\mathbf{C 2}$ are correctly formed, then the KRA can first recover $\psi^{k}$ from $A$, then $x_{i}^{-1}$ and thus $x_{i}$ from $B_{i}$ $(i=s-1, \ldots, 0)$. The transcript in C3, consists of two parts. The first part is the $(t-s)$-tuple

$$
\left(d_{t-1}, d_{t-2}, \ldots, d_{s}\right)=\left(g^{\left[\psi^{k}\right]_{t-1}}, g^{\left[\psi^{k}\right]_{t-2}}, \ldots,\left(g^{\left[\psi^{k}\right]_{s}}\right)\right.
$$

The second part is a transcript of a non-interactive proof that there exists a $0<k<\omega$ such the following two equalities in power polynomials hold:

$$
\begin{aligned}
g^{\left(\psi^{k}\right)}= & d_{t-1} X^{t-1}+d_{t-2} X^{t-1}+\ldots+d_{s} X^{s}+ \\
& +y_{s-1}^{B_{s-1}} X^{s-1}+y_{s-2}^{B_{s-2}} X^{s-2}+\ldots+y_{0}^{B_{0}} \\
\gamma^{k}= & A
\end{aligned}
$$


If the above two equalities hold for some $k$, then this shows that $\mathbf{C 1}$ and $\mathbf{C 2}$ are correctly formed, i.e. contain the same $x_{i}$ for $i=s-1, \ldots, 0$. It is clear from Section 3 how to form the transcript in the schemes as mentioned in Scheme 41. With respect to the choice of the security parameter $v, 2^{-60}$ seems like a reasonable probability of failure. Moreover, certification is usually an on-line process, in which a users authenticates himself using an access code given by an RA. Hence one can easily incorporate an online challenge of the Certification Authority in the input of the hash resulting in the challenges $h_{1}, \ldots, h_{v}$, and puts a maximum time on the certification session, then $2^{40}$ seems like a reasonable bound for $K$. This means that $v=100$ seems like a reasonable choice for the security parameter.

As an illustration, suppose that $G$ is a group consisting of a collection of points on an elliptic curve of prime order $p$ of 160 bit length, then one can compare this with the security of the ElGamal encryption system in the multiplicative group of a basic field of size 1600 bits, cf. [14]. So to get the same level for security for the KRA - as in the construction of CRs in [27] - one could employ our subgroup variant in $G F\left(p^{t}\right)$ with $t=10$. This would result in a CR of about 64,000 bits, much smaller than the length of a comparable CR in the schemes in [27], which would be of size 160,000 bits. Observe that $t=10$ also facilitates the use of optimal normal bases (as mentioned in Section 2.2), making our scheme also more efficient than comparable schemes in [27]. To get more security for the KRAs, say "1900 bits", one could use a similar technique in $G F\left(p^{t}\right)$ with $t=12$. This would then still result in a CR of 64,000 bits, still smaller than the length of the above mentioned transcript.

\subsection{Security Analysis of our Scheme}

With respect to security, let us first look at Scheme 41 with $s=t$. Then the first part of component C3 (mentioned in formula (5)) is empty, and it follows that C3 is a transcript of a proof of knowledge from which no secret information can be extracted. Now, an attacker could try to find one of the user's private keys, $x_{i}$ say, using one the following three strategies. First, he could try to find $x_{i}$ from component $\mathbf{C 1}$, which would mean breaking the discrete logarithm in $G$.

Secondly, he could try to find $x_{i}$ from component C2, which would mean breaking the Diffie-Hellman problem in $\Gamma$ by Corollary 22. This problem is designed to be more difficult (in a scalable fashion) than to break the public key of the user.

Finally, he could try to find $x_{i}$ from combining the information from the components $\mathbf{C 1}$ and $\mathbf{C 2}$. As the discrete logarithm problems employed in $\mathbf{C 1}$ and $\mathbf{C 2}$ are only related by the number $p$, it seems unlikely that combining both components $\mathbf{C 1}$ and $\mathbf{C 2}$ will be very beneficiary. We'll now briefly discuss a formal result in this direction.

We recall from Section 2.1 that a suitable generator $\gamma$ of the multiplicative group of $G F\left(p^{t}\right)$ should have a prime subgenerator that is not contained in one of the proper subfields and should have a suitably large prime order $\varpi$. 
In our constructions, it is very likely that the power of $\varpi$ in the primenumber decomposition of $p^{t}-1$ is one, i.e. $p^{t}-1 / \varpi$ and $\varpi$ are relatively prime. One can uniquely decompose $\gamma$ as the product $\gamma^{\prime} \cdot \gamma^{\prime \prime}$ where the order of $\gamma^{\prime}$ equals $\varpi$ and that of $\gamma^{\prime \prime}$ equals $p^{t}-1 / \varpi$.

The following result is a generalization of [24, Proposition 1].

Theorem 42 We use the above notation. Let the ElGamal encryptions used for the KRA be classical, i.e. the generator $\gamma$ generates the whole multiplicative group of $G F\left(p^{t}\right)$ and let $i$ be one of $0, \ldots, t-1$. Also, let the power of $\varpi$ in the primenumber decomposition of $p^{t}-1$ be one.

Then, using an efficient algorithm $\mathcal{P}$ computing $x_{i}$ from $\mathbf{C 1}$ and $\mathbf{C 2}$, one can either construct

1. an efficient algorithm $\mathcal{P}_{\mathcal{D} \mathcal{L}}$ solving the discrete logarithm problem in $G$, or

2. an efficient algorithm $\mathcal{P}_{\mathcal{D D} \mathcal{H}}$ solving the Decision-Diffie-Hellman problem w.r.t. the base $\gamma^{\prime}$.

Sketch of Proof: Suppose that $\mathcal{P}$ is an efficient algorithm computing $x_{i}$ from C1 and C2. Consider a triple $(A, B, C)$ in $\left\langle\gamma^{\prime}\right\rangle^{3}$. Using the technique appearing in the proof of [24, Proposition 1], one can transform this to a random triple $(\hat{A}, \hat{B}, \hat{C})$ in $\left\langle\gamma^{\prime}\right\rangle^{3}$ that is either a random Diffie-Hellman triple if $(A, B, C)$ is, or a random non-Diffie-Hellman triple otherwise.

Moreover, by suitably multiplying $\hat{A}, \hat{B}, \hat{C}$ with random Diffie-Hellman triples in $\left\langle\gamma^{\prime \prime}\right\rangle^{3}$, one obtains a triple $(\bar{A}, \bar{B}, \bar{C})$ in the multiplicative group of $G F\left(p^{t}\right)$, that is either a random Diffie-Hellman triple if $(A, B, C)$ is, or a random nonDiffie-Hellman triple, with respect to the element $\gamma^{\prime}$ otherwise. Hence, $(\bar{A}, \bar{B}, \bar{C})$ is always a random Diffie-Hellman triple with respect to $\gamma^{\prime \prime}$.

Using the technique appearing in the proof of [24, Proposition 1], one can now either construct

1. an efficient algorithm $\mathcal{P}_{\mathcal{D} \mathcal{L}}$ solving the discrete logarithm problem in $G$, or

2. an efficient algorithm $\mathcal{P}_{\mathcal{D} \mathcal{D} \mathcal{H}\left(\gamma^{\prime}\right)}$ solving the Decision-Diffie-Hellman problem w.r.t. the base $\gamma^{\prime}$.

Let us assume that the DH decision problem in $\left\langle\gamma^{\prime}\right\rangle$ is as hard as the DL problem in $\left\langle\gamma^{\prime}\right\rangle$, which is constructed to be more difficult than the DL problem in $G$. Then we can conclude from the previous result that the best strategy an attacker can follow to determine $x$ from $\mathbf{C 1}$ and $\mathbf{C 2}$ is to break the DL problem in $G$. Hence, at least from a theoretical point of view, using the classical ElGamal scheme in our scheme is more secure than using the subgroup variant. However, we do not expect that using the subgroup variant of the ElGamal in our scheme is less secure in practice than using the classical ElGamal scheme.

We are left with a general security proof of the Scheme 41, let $s=s_{0}$ with $1 \leq s_{0}<t$. Now, the idea is that we will construct an arbitrary "output" of our scheme with $s=s_{0}$ from an arbitrary "output" with $s=t$. So, if one has an 
advantage in breaking the scheme with $s=s_{0}$, one also has this advantage for breaking the scheme with $s=t$. As we have shown the security for the scheme with $s=t$ this would conclude the security proof of the scheme with $s=s_{0}$ also.

For this construction, let C1, C2 and C3 be an arbitrary output for the scheme with $s=t$. Forming C1' and C2' for the scheme with $s=s_{0}$ simply means removing some information from C1 and C2. Also, from the transcript in C3' one obtains a representation of the power polynomial $g^{\left(\psi^{k}\right)}$ (see equality (6)). So we can form the first part $\left(d_{t-1}, d_{t-2}, \ldots, d_{s}\right)$ of C3' by simply taking the highest $t-s$ coefficients of the power polynomial $g^{\left(\psi^{k}\right)}$. Finally, the second part of C3' must be a random transcript that proves that equality (6) holds. However, the second part of $\mathbf{C 3}$ provides us with such a random transcript.

\subsection{Improvements and Generalizations of our Scheme}

Reduced Ciphertext In our basic scheme, the user has total freedom in choosing his private keys which might be useful in some situations (e.g. when using already existing public keys). However, if there are no restrictions on the user's private keys other than that they should be uniformly random, then the component $\mathbf{C}_{2}$ can be reduced, like is done in [27]. Let us assume that the number of user public keys equals the extension degree of the field, i.e. $s=t$; the case $s<t$ is a straightforward generalization.

In this situation, the user first generates $\gamma^{k}$ where $0<k<\omega$ is randomly chosen and then chooses the private $x_{i}$ as $\left[\psi^{k}\right]_{i}$, and forms his public keys as $y_{i}=g^{x_{i}}$ as usual $(i=0, \ldots, s-1)$. The user then forms components $\mathbf{C}_{\mathbf{1}}$ and $\mathbf{C}_{\mathbf{2}}$, the latter of which only needs to contain $\gamma^{k}$ as $x_{i}^{-1} \cdot\left[\psi^{k}\right]_{i}=1$. The component $\mathbf{C}_{\mathbf{3}}$ is formed as before. Note the following equality for power polynomials holds (modulo the fixed irreducible polynomial $F$ ):

$$
g^{\left(\psi^{k}\right)}=y_{t-1} X^{s-1}+y_{s-2} X^{s-2}+\ldots+y_{0}
$$

That is, the $t$ public keys of the user can be represented by the power polynomial $g^{\left(\psi^{k}\right)}$. Hence, the components $\mathbf{C}_{\mathbf{1}}, \mathbf{C}_{\mathbf{2}}$ in the improved scheme, simply take the form

$$
g^{\left(\psi^{k}\right)}, \gamma^{k}
$$

And $\mathbf{C}_{\mathbf{3}}$ is a non-interactive proof showing that $k$ occurs in both components. It easily follows that this improved scheme is a secure as the basic one. and that the output of the improved scheme is $s \cdot \omega$ bits smaller than the basic scheme.

Reduced Risk of Shadow Key Establishment It is not hard to leak 20 bits of information in each of the $y_{i}$ appearing in the component $\mathbf{C}_{\mathbf{1}}$ in the basic scheme as they can be independently chosen. So, if $t=12$ then this would mean that 240 bits subliminal bits can be leaked in the component $\mathbf{C}_{\mathbf{1}}$, which opens the possibility of the establishment of shadow public keys. Of course, this problem arises with any private key escrow scheme where the user can construct 
his public key himself and which allows for several, say 12, (certified) public keys to be used. Nonetheless, using the above described improved scheme reduces this risk, as the user public keys are no longer generated independently.

Incorporating Secret Sharing In all our schemes so far, we have used a public key $\psi$ of which the private part $x_{K}$ was in the possession of only one Key Recovery Agent. It is well-known that Scheme 41 can very conveniently support the use of a KRA's public key in which the private key is secretly shared among $n$ share-holders, i.e. sub-KRAs. For instance, suppose that all sub-KRAs have chosen a private key $0<\theta_{i}<\omega$, and a public key $\psi_{i}=\gamma^{\theta_{i}}$. Then the product $\psi$ of the latter will be the shared KRA public key; it easily follows that private keys of users can be reconstructed without the sub-KRAs having to come together. Indeed, the $i$-th sub-KRA forms (using the terminology from Scheme 41) $A_{i}=A^{\theta_{i}}$ and sends this to the entity requesting recovery of the user's private keys. Of course, the privilege and execution of this should be in accordance with the key recovery policy. This entity forms the product of these $A_{i}$ which equals $\psi^{k}$ from which the user's private keys can be retrieved.

In the subgroup variant of our schemes where $\omega$ is a prime number, further convenient improvements are possible. Here participating sub-KRA's can, without needing a trusted dealer, for any $1 \leq k \leq n$, construct a public key $\psi$ in such a way that if it is used in our basic scheme, then the user's private key can be reconstructed only if $k$ out of $n$ KRA sub-holders cooperate. See [20] and [21]. Note that if one wants to use this technique to implement a $k$ out of $n$ key recovery scheme, then it is required that the sub-KRAs generate $\left(\begin{array}{l}n \\ k\end{array}\right)$ public keys, which grows exponentially in $k$.

An improvement by Schoenmakers in [22] solves a similar problem in the original CR scheme of Young and Yung [27]. However, this improvement still has the drawbacks mentioned in the introduction, specifically the lack of scalable security. By combining the techniques in [22] with ours, we can resolve these drawbacks.

More specifically, this combination offers:

- scalable security,

- the possibility that users themselves can choose a number, $n$, of KRAs from a given list consisting of $l$ KRAs $(n \leq l)$, and can choose a number $k \leq n$ such that $k$ out of $n$ of the chosen KRAs are required to retrieve the users private keys,

- the ability for users to form Certificates of Recoverability proving the correctness of the previous point to the CA.

Of course, the choice of $k$ and $n$ the user makes should be in accordance with the Certificate Policy under which the CA operates: if this choice is not acceptable, then the CA will not issue an certificate.

To this end, without loss of generality we again assume that $s=t$. Using the technique of [22] in our schemes, means that the private part, i.e. $\log _{\gamma}(\psi)$ of the 
public key $\psi$ is "virtual", i.e. it is not known by anybody, and that all involved parties are certain of that. Or, in other words, $\gamma$ and $\psi$ are independently selected generators. Moreover, each $i$-th sub-KRA has a public key $\xi_{i}$, with respect to $\psi$ (and not to $\gamma$ as before), i.e. $\xi_{i}=\psi^{\theta_{i}}$ where $\theta_{i}$ is the private key of the sub-KRA. Now, the user encrypts his private keys using the improved scheme described above using the virtual public key $\psi$. As mentioned above the components $\mathbf{C}_{\mathbf{1}}$, $\mathbf{C}_{2}$ then take the form

$$
g^{\left(\psi^{k}\right)}, \gamma^{k}
$$

And $\mathbf{C}_{3}$ is a non-interactive proof showing that $k$ occurs in both components. Of course, as $\psi$ is a virtual public key, nobody is able to retrieve the user's private keys from these components. This is why the user supplements these components by using the non-interactive Public Verifiable Secret Sharing scheme in [22], secretly sharing $\psi^{k}$ among the KRA's, using the public keys $\xi_{i}$ where $\gamma^{k}$ is used as the initial commitment $\left(C_{0}\right.$ in the terminology of [22]). We refer to [22] for further technical details. One can easily prove that this supplementation to our schemes does not weaken security.

\section{Conclusion}

We have proposed two schemes for Certificates of Recoverability, making it possible for a PKI user to escrow its private keys in a publicly verifiable way, by means of encrypting it with a Key Recovery Agent's public key and depositing this with any other party. In our schemes, the cryptographic security employed by the Key Recovery Agents can be set higher, in a scalable fashion, than that being employed by the users. Among the other improvements of our scheme are its applicability to create CRs for cryptosystems based on the Discrete Log problem in small subgroups such as Elliptic Curve Cryptosystems. Also, the size of the constructed proofs of knowledge can be taken smaller. We have additionally shown several ways to support secret sharing in our scheme. Finally, we have also presented several new constructions and results on the hardness of "small parts", in the setting of Diffie-Hellman keys in extension fields.

\section{Acknowledgements}

Berry Schoenmakers and an anonymous referee are gratefully acknowledged for their constructive comments.

\section{References}

1. M. Adleman, J. DeMarrais A subexponentional algorithm over all finite fields, CRYPTO '93 Proc., Springer-Verlag, 147-158. 262

2. N. Asokan, V. Shoup, M. Waidner, Optimistic Fair Exchange of Digital Signatures, Eurocrypt'98 Proc., Springer-Verlag, 591-606. 260

3. M. Bellare, P. Rogaway, Random Oracles are Practical: A paradigm for Designing Efficient Protocols, 1st ACM Conference on Computer and Communications Security, ACM Press, 1993, 62-73. 268 
4. D. Boneh, R. Venkatesan, Hardness of Computing the Most Significant Bits of Secret Keys in Diffie-Hellman and Related Schemes, CRYPTO'96 Proc. SpringerVerlag, 129-142. 263

5. A.E. Brouwer, R. Pellikaan, E.R. Verheul, Doing More with Fewer Bits, Asiacrypt'99 Proc., Springer-Verlag. 266

6. D. Coppersmith, Fast evaluation of logarithms in fields of characteristic two, IEEE Trans. on IT, 30, 1984, 587-594. 262

7. W. Diffie, M.E. Hellman, New directions in cryptography, IEEE Trans. on IT 22, 1976, 644-654. 261

8. T. ElGamal, A Public Key Cryptosystem and a Signature scheme Based on Discrete Logarithms, IEEE Trans. on IT 31(4), 1985, 469-472. 259, 261

9. A. Fiat, A. Shamir, How to prove yourselve: Practical solutions to identification and signature problems, CRYPTO'86 Proc., Springer-Verlag, 186-194. 268

10. D.M. Gordon, Discrete Logarithms in $G F(p)$ using the number field sieve, SIAM J. of Discrete Math., 6, 124-138. 260

11. J. Håstad, On Using RSA with Low Exponent in a Public Key Network, CRYPTO'85 Proc., Springer-Verlag, 403-405. 259

12. J. Kilian, F.T. Leighton, Fair Cryptosystems Revisited, Crypto'95 Proc., SpringerVerlag, 208-221. 261

13. A.K. Lenstra, Using Cyclotomic Polynomials to Construct Efficient Discrete Logarithm Cryptosystems over Finite Fields, ACISP97 Proc., Springer-Verlag, 127-138. 262, 263, 265

14. A.K. Lenstra, E.R Verheul Selecting Cryptographic Key Sizes, these proceedings. 270

15. H.W. Lenstra, Finding isomorphisms between two finite fields Math. of Comp., 56 (1991), 329-347. 266

16. R. Lidl, H. Niederreiter, Finite Fields, Addison-Wesley, 1983. 264

17. S.C. Pohlig, M.E. Hellman, An improved algorithm for computing logarithms over $G F(p)$ and its cryptographic significance, IEEE Trans. on IT, 24 (1978), 106-110. 262

18. J.M. Pollard, Monte Carlo methods for index computation ( $\bmod p$ ), Math. of Comp., 32 (1978), 918-924. 262

19. M. Naor, M. Yung, Universal one-way functions and their cryptographic applications, In 21st Annual ACM Symposium on Theory of Computer Science, 1997. 266

20. T.P. Pedersen, Distributed Provers with Applications to Undeniable Signatures, Eurocrypt'91 Proc., Springer-Verlag, 221-242. 273

21. T.P. Pedersen, A Treshold Cryptosystem Without a Trusted Party, Eurocrypt '91 Proc., Springer-Verlag, 522-526. 273

22. B. Schoenmakers, A Simple Publicly Verifiable Secret Sharing Scheme and Its Application to Electronic Voting, CRYPTO'99 Proc., 148-164. 273, 274

23. C. Schnorr, Efficient signature generation by smart cards, Journal of Cryptology, 4, 1991, 161-174. 260

24. M. Stadler, Publicly Verifiable Secret Sharing, Eurocrypt'96 Proc., 190-199. 259, 261, 267, 271

25. M. Stadler, J.-M. Piveteau, J. Camenisch, Fair Blind Signatures, Eurocrypt'95 Proc., 209-219. 261

26. D.R. Stinson Cryptography: theory and practice, CRC press, 1995. 259, 260

27. A. Young, M. Yung, Auto-Recoverable Auto-Certifiable Cryptosystems, Eurocrypt'98 Proc., 16-31. 259, 260, 261, 268, 270, 272, 273 